\title{
Numerical Study of Fluidic Thrust Vector Control using Dual Throat Nozzle
}

\author{
K. X. Wu, T. H. Kim and H. D. Kim ${ }^{\dagger}$ \\ Mechanical Engineering, Andong National University, Andong, 760-749, South Korea \\ †Corresponding Author Email: kimhd@anu.ac.kr
}

(Received April 8, 2020; accepted June 16, 2020)

\begin{abstract}
Compared to a variety of mechanical vectoring nozzles, fluidic vectoring nozzles possess more research value nowadays. The dual throat nozzle is gradually developing into an outstanding technology to handle supersonic and hypersonic aircraft deflections. Three-dimensional, steady, compressible, and viscous flows in rectangular dual throat nozzles are numerically investigated by resolving Reynolds-averaged Navier-Stokes equations and shear stress transport $k$-omega turbulence model. Computational fluid dynamics results are verified against the existing experimental data, where a good consistency is gained. The impacts of nozzle pressure ratio, injection-to-mainstream momentum flux ratio, and setup angle of the slot injector on the systemic performance are examined. Useful conclusions are summarized for engineering designers. Firstly, pitching angles decline along with an increasing nozzle pressure ratio, while systemic thrust ratio and thrust efficiency increase. Secondly, thrust vector angles enlarge with an increase of the injection-to-mainstream momentum flux ratio, whereas both systemic thrust ratio and thrust efficiency decay. Finally, the setup angle of the slot injector impacts the systemic performance remarkably. Although the pitching angle for the setup angle of $120^{\circ}$ is highest, comprehensive characteristics in terms of systemic thrust efficiency and systemic thrust ratio for the setup angle of $150^{\circ}$ are more excellent.
\end{abstract}

Keywords: Vector control; Jet deflection; Shock wave; Compressible flow; Internal flow.

\section{NOMENCLATURE}

$A_{e} \quad$ nozzle exit area

$C_{r} \quad$ systemic thrust efficiency

$C_{s} \quad$ systemic thrust ratio

$D \quad$ width of the slot injector

$F_{i} \quad$ ideal thrust

$F_{i, m}$ ideal mainstream thrust

$F_{i, s} \quad$ ideal secondary stream thrust

$F_{x} \quad$ horizontal thrust

$F_{y} \quad$ vertical thrust

$F_{z} \quad$ lateral thrust

$H_{u t} \quad$ upstream minimum throat height

$H_{d t} \quad$ downstream minimum throat height

$I P R$ injection pressure ratio

$J \quad$ injection-to-mainstream momentum flux ratio

$L \quad$ length of the slot injector

$L_{c} \quad$ length of the concave cavity

$m_{0} \quad$ mass flow rate of the mainstream

$m_{i} \quad$ mass flow rate of the secondary stream

$N P R$ nozzle pressure ratio

$P \quad$ static pressure

$P_{0} \quad$ total pressure of the nozzle inlet
$P_{i} \quad$ total pressure of the slot inlet

$P_{\text {atm }}$ standard ambient pressure

$P_{e} \quad$ weighted average pressure in the nozzle exit plane

$P_{u w} \quad$ static pressure along the centerline on the upper cavity wall

$R_{g} \quad$ specific gas constant

$T$ static temperature

$T_{0}$ total temperature

$V \quad$ velocity

$V_{e x} \quad \mathrm{X}$-component of the nozzle exit velocity

$V_{e y} \quad$ Y-component of the nozzle exit velocity

$V_{e z} \quad$ Z-component of the nozzle exit velocity

$W \quad$ nozzle width

$\xi \quad$ injection-to-mainstream mass flow ratio

$\gamma \quad$ specific heat ratio $(\gamma=1.4$ for air)

$\rho$ density

$\delta_{\beta} \quad$ pitching angle

$\theta_{1} \quad$ divergence angle

$\theta_{2}$ convergence angle 


\section{INTRODUCTION}

Nowadays, the thrust vector control (TVC) quickly develops as an advanced and efficient fluid control technology, which supplies pitching, rolling, and yawing momentums for supersonic and hypersonic aircraft, as shown in Fig. 1 (Wu et al. 2020a). Fig. 2 shows the classification of the TVC, thereinto, one is fluidic TVC (FTVC) and the other is mechanical TVC (MTVC). The MTVC generally relies on mobile jet vanes and nozzles to finish particular flight paths (Fleeman 2006). Outstanding superiority is its reliability, whereas many mechanical components result in redundant complexity and weight (Sutton and Biblarz 2001). However, the FTVC implements the same control effect within a much lower weight and cost (Das et al. 2016). Wu et al. (2020a) declared that a supersonic or hypersonic aircraft owing FTVC can thoroughly gain the benefit of airfield or carrier runway independence through vertical ascent and landing.

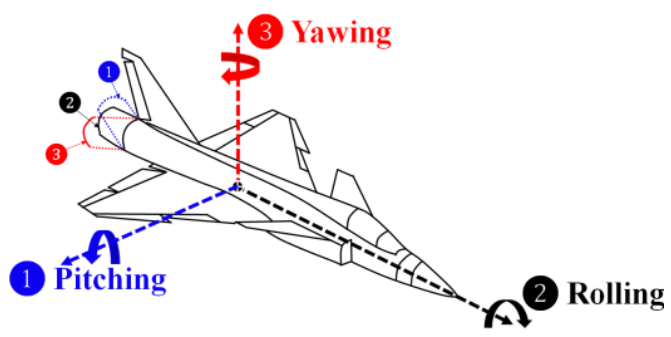

Fig. 1. Illustration of aircraft axes.

A few of fluidic vectoring concepts and principles have been proposed and listed in Fig. 2, e.g., shock wave TVC (SWTVC), bypass shock wave TVC (BSWTVC), counterflowing TVC (COUTVC), coflowing TVC (COTVC), throat shifting TVC (TSTVC), dual throat nozzle TVC (DTNTVC), and bypass dual throat nozzle TVC (BDTNTVC). Wu and Kim (2019a; 2019b) and Wu et al. (2020c) carried through theoretical and computational fluid dynamics (CFD) work about steady features of the SWTVC in a supersonic rectangular propulsion nozzle and detailedly illuminated the impacts of injection-to-mainstream momentum flux ratio, injector location, and the length-to-width ratio of the slot injection syringe. Deng and Kim (2015) finished some CFD work concerning internal flow characteristics of the BSWTVC and elucidated the influences of nozzle pressure ratio (NPR) and injection-to-mainstream mass flow ratio, separately. Notwithstanding SWTVC and BSWTVC technologies can come true high vectoring angles, induced oblique and bow shock waves affect the systemic thrust ratio prominently (Wu and Kim 2019b; Deng and Kim 2015). Both COUTVC and COTVC have gained much interest because of their large jet deflections and less blowing or suction mass flow (Heo and Sung 2012; Wu et al. 2020a; Wu et al. 2020d; Kim et al. 2020; Wu et al. 2018). Nevertheless, some tough issues still need to be well addressed, specifically, the hysteresis impact and the source of the blowing and suction streams
(Wu et al. 2019).

Deere (2003) and Deere et al. (2003) carried through experimental and CFD analyses on TSTVC and expounded that the highest systemic thrust ratio of around 0.96 is available. Yagle et al. (2001) and Mier et al. (1999) experimentally found that measured vectoring angles are always lower than those of the SWTVC. The DTNTVC resolves a few shortcomings of the TSTVC and therefore is particularly studied. It is worth highlighting that it has larger jet deflections and systemic thrust efficiencies without compromising the systemic thrust ratio as well. The working principle of DTNTVC is drawn in Fig. 3, where a convergentdivergent-convergent (C-D-C) nozzle configuration with two geometric minimum throat areas is utilized. A concave cavity, therefore, forms between two minimum geometry throats. One asymmetric injector is located at the upstream nozzle throat with an adaptive setup angle (Note that: another injector is necessary to achieve an opposite mainstream deflection in realistic applications). Since the secondary stream is injected at the upstream throat asymmetrically, the flow separation appears and the skew sonic-plane occurs, simultaneously.

During the past decade, some researchers finished experimental and CFD work and offered some worthy conclusions. Ferlauto and Marsilio (2009) testified that the DTNTVC has a comprehensive performance improvement than that of the TSTVC. To better perform a variety of operating situations, Deere et al. (2007) and Flamm et al. (2007) computationally and experimentally examined the impact of several nozzle design variables on the internal performance of a conical DTNTVC, e.g. divergence or convergence angle of the concave cavity, scope of circumferential injection, and cavity length. Wu and Kim (2019c), Bellandi and Slippey (2009), and Shin et al. (2010) numerically argued that NPR plays a critical role in a twodimensional (2-D) DTNTVC. Flamm et al. (2006) experimentally and computationally studied the impact of geometric structure variables in a threedimensional (3-D) rectangular DTNTVC system, e.g. convergence or divergence angle of the cavity, injector type, and cavity shape. They demonstrated that the slot injector can obtain larger jet deflections than a hole injector. Since the starting issues of axisymmetric divergent dual-throat nozzles cause a lot of thrust losses, Wang et al. (2017) devoted themselves to studying both steady and unsteady characteristics in this specific process. They elucidated that the shock oscillation appears inside the concave cavity under some high nozzle expansion ratios and expounded the influences of rounding radius of the nozzle throat and cavity bottom, cavity convergence angle, cavity length on its systematic performance. To pursue higher thrust efficiencies, a new fluidic vectoring nozzle was developed by adding a bypass, that is to say, BDTNTVC. Gu et al. (2014; and Gu and Xu 2015) carried through experimental and CFD work about steady and unsteady features of the BDTNTVC and clarified that it improves the thrust efficiency in comparison to that of the DTNTVC. However, only 


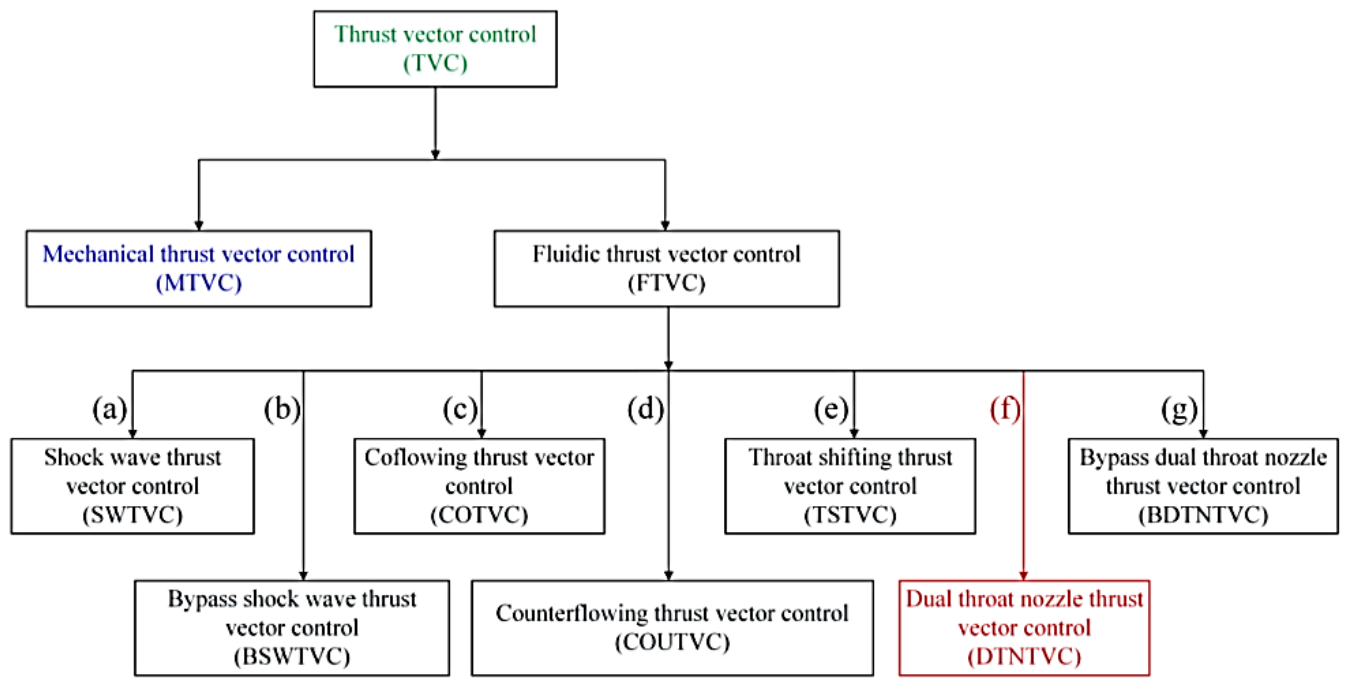

Fig. 2. Classification of the TVC.

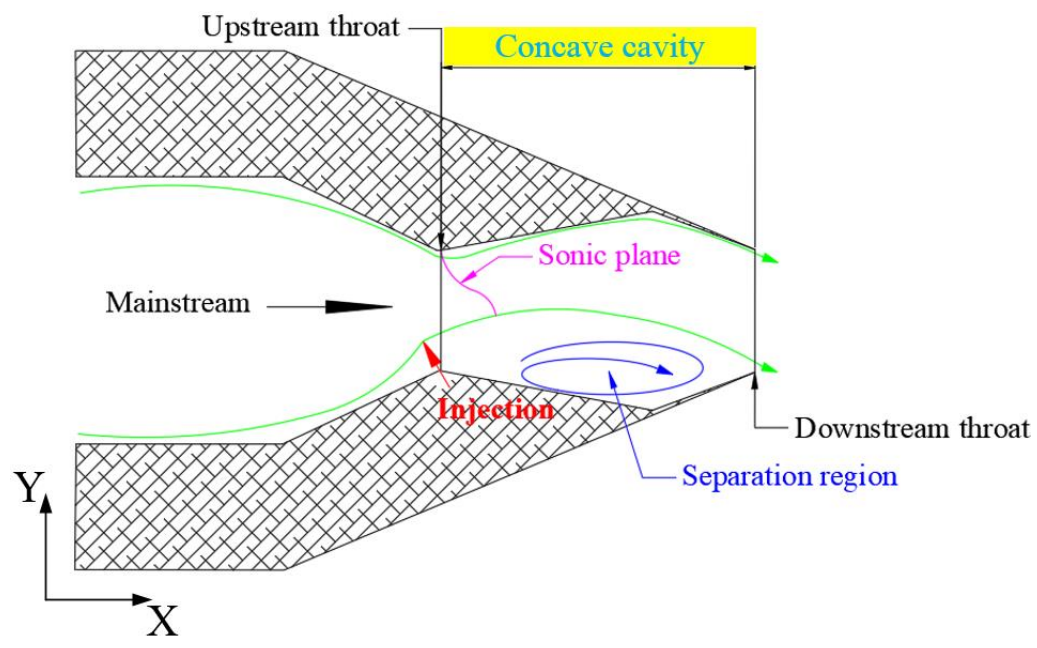

Fig. 3. Illustration of the DTN.

one control parameter, NPR, can be manipulated to handle an effective vectoring control. Thus, the flexibility and effectiveness of the BDTNTVC are severely limited, which should be further resolved to better adapt to engineering implementations (Wang et al. 2019a).

The motivations of the current study are summed up herein: Firstly, supersonic rectangular nozzles have prominent advantages in terms of stealth and infrared characteristics. They have been used in many real supersonic fighters, such as F-22 Raptor, F-15S/MTD, and SU-27 Flanker. Wang et al. (2019b) experimentally confirmed the real flow features in the conical DTN are not fully axisymmetric based on oil flow visualization technology. It is not well demonstrated that if this asymmetric flow characteristic would occur in a 3D rectangular DTN. As a consequence, a 3-D rectangular DTNTVC is investigated here. Secondly, earlier studies were concentrated on different geometry parameters of the concave cavity. To pursue the design goal of most efficient control effectiveness in the preliminary stage, not only cavity structure but also pressure control needs to be optimized. Very few investigations have been carried through to expound the impacts of NPR and injection-to-mainstream momentum flux ratio on the spatial development of flow-field in a 3-D rectangular DTNTVC system. As a result, it is necessary to demonstrate their impacts on systemic thrust performance. Finally, the influence of the setup angle of the slot injection syringe on systemic performance is elucidated to seek out the most suitable scheme.

\section{DeFinition OF ASSESSMENT PARAMETERS}

The pitching angle, $\delta \beta$, is derived from the ratio of thrust along the vertical direction (Y-direction) and the horizontal direction (X-direction), as shown in the following Eqs. (1-3).

$$
\begin{aligned}
\delta_{\beta} & =\tan ^{-1}\left(F_{y} / F_{x}\right) \\
F_{x} & =\left(m_{0}+m_{i}\right) V_{e x}+\left(P_{e}-P_{a t m}\right) A_{e}
\end{aligned}
$$




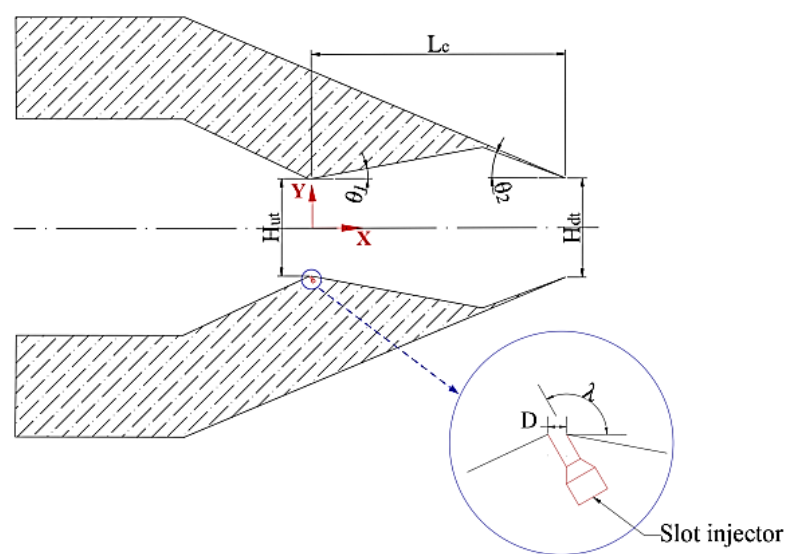

Fig. 4. Dimensions of the DTN.

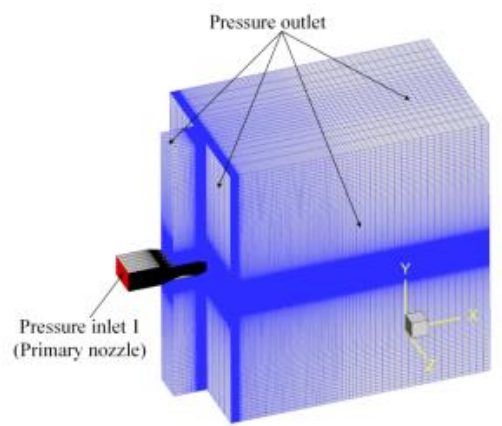

(a)

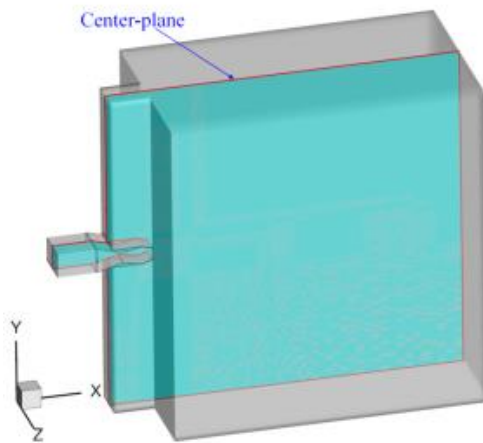

(c)

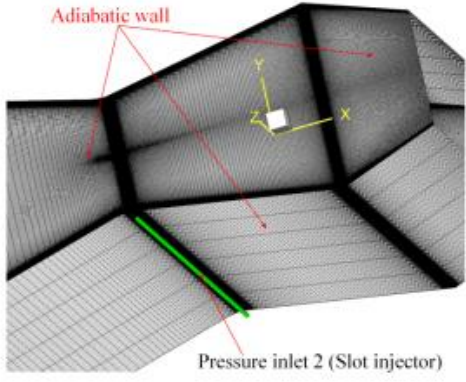

(b)

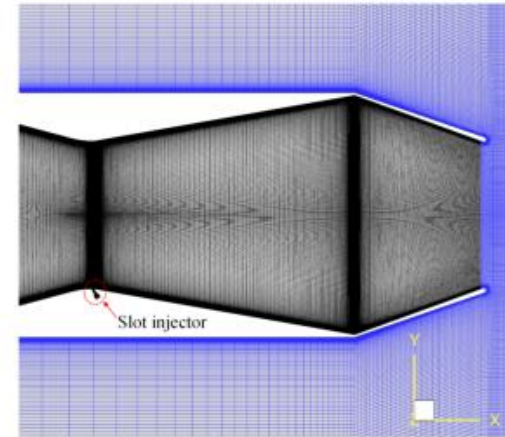

(d)

Fig. 5. Computational grid and boundary conditions (a) full-domain; (b) partial domain; (c) centerplane $(Z=0)$; (d) grid on the center-plane.

$F_{y}=\left(m_{0}+m_{i}\right) V_{e y}$

$F_{Z}=\left(m_{0}+m_{i}\right) V_{e z}$

Where Fx, Fy, and Fz are horizontal, vertical, and lateral components of the thrust. Vex, Vey, and Vez are horizontal, vertical, and lateral components of the exit velocity. $A_{e}$ denotes the exit area of the DTN. mi and $\mathrm{m} 0$ represent mass flow rates of the secondary stream and the mainstream, severally. Pe is the weighted average pressure in the DTN exit plane, and Patm represents the ambient pressure.

The definition of the injection-to-mainstream mass flow ratio, $\alpha$, is given by the following Eq. (5).

$\alpha=m_{i} / m_{0}$
The systemic thrust ratio, Cs, is defined as the ratio of realistic and ideal thrust, as shown in Eq. (6).

$C_{S}=\frac{\sqrt{F_{x}{ }^{2}+F_{y}{ }^{2}+F_{z}^{2}}}{F_{i}}$

The ideal thrust, Fi, is made up of two portions that are the ideal mainstream thrust, Fi,m, and the ideal thrust of the secondary stream, Fi,s.

$F_{i}=F_{i, m}+F_{i, s}$

$F_{i, m}=m_{0} \sqrt{\frac{2 \gamma R_{g} T_{0}}{\gamma-1}\left[1-\left(\frac{1}{N P R}\right)^{\frac{\gamma-1}{\gamma}}\right]}$

$F_{i, s}=m_{i} \sqrt{\frac{2 \gamma R_{g} T_{0}}{\gamma-1}\left[1-\left(\frac{1}{I P R}\right)^{\frac{\gamma-1}{\gamma}}\right]}$ 
The systemic thrust efficiency, $\mathrm{Cr}$, is deduced from the pitching angle and the injection-to-mainstream mass flow ratio, as given in the following Eq. (10).

$C_{r}=\frac{\left|\delta_{\beta}\right|}{\left(m_{i} / m_{0}\right) * 100}$

The injection-to-mainstream momentum flux ratio, $\mathrm{J}$, is formulated in Eq. (11).

$J=\frac{\rho_{i} u_{i}^{2}}{\rho_{m} u_{m}^{2}}=\frac{\gamma_{i} p_{i} M_{i}^{2}}{\gamma_{m} p_{m} M_{m}^{2}}$

\section{Numerical Procedure}

\subsection{Numerical Setup}

Fig. 4 displays the current DTN geometry, which is referred to previous experimental work of Flamm et al. (2006). Downstream and upstream nozzle throat heights are the same, in which Hdt $=$ Hut $=0.0292$ $\mathrm{m}$. The length of the concave cavity is $\mathrm{Lc}=0.0762$ $\mathrm{m}$, and its width is $\mathrm{W}=0.1016 \mathrm{~m}$. The divergence and convergence angles of the concave cavity are $\theta 1=10^{\circ}$ and $\theta 2=20^{\circ}$. The slot injection syringe is situated at the bottom of the upstream nozzle throat. The width of the slot injection syringe is $\mathrm{D}=0.0005$ $\mathrm{m}$, the length is $\mathrm{L}=0.1016 \mathrm{~m}$, and the setup angle is $\lambda=120^{\circ}$.

Fig. 5 shows a full computational field and necessary boundary conditions. The field has a size of $28 \mathrm{Hut} \times 14 \mathrm{Hut} \times 7 \mathrm{Hut}$ along with horizontal, vertical, and lateral directions. Structured grids are used to catch the 3-D flow-field features precisely. The mesh refinement is carried through in the neighborhood of the upstream and downstream nozzle throats and the divergence-convergence (DC) junction of the concave cavity, as shown in Fig. 5(d). Concerning the viscous effect, the boundary layer grids are made along with nozzle surfaces. The maximum value of wall $y+$ is 0.95 . The pressure inlet boundary condition is adopted for both entrances of the nozzle and the slot injection syringe. All nozzle and slot injection syringe surfaces are assumed as adiabatic walls. Other exit boundaries are taken as the pressure outlet boundary.

All current CFD work is finished in the software of Fluent that is good at calculating incompressible and compressible flows based on pressure-based and density-based solvers (Lin et al. 2020; Tao et al. 2020; Wang et al. 2019a; Wang et al. 2019b). By referring to earlier references (Deng and Kim 2015; Vignesh et al. 2016; Vignesh et al. 2019; Wu and Kim 2019a; Raman et al. 2020; Wu et al. 2020a), the density-based solver is fixed for issues of compressible flow. The implicit formulation is used to address the equation system. The working fluid is an ideal gas and the gas viscosity is addressed with the law of Sutherland. The AUSM is chosen to solve conservation equations. The spatial discretization gradient uses the least square cellbased methodology with the specific scheme of the second-order upwind.

\subsection{Grid Independence Analysis}

A grid independence analysis is executed among three different grid systems. Fig. 6 shows static pressure distributions along the center-plane of the upper cavity wall for three grid systems. The grid cells involve a coarse grid 1 of 1.036 million cells, a medium grid 2 of 2.078 million cells, and a fine grid 3 of 4.121 million cells. The result of grid 1 shows a certain differential in comparison to the other two grids. Static pressure distributions of grid 2 and grid 3 are very close. Further, a grid convergence index mentioned by Roache (1994) is calculated. Three grids have a refinement ratio $r$ of 2 inside this concave cavity. The maximum pressure ratio is chosen as the convergence parameter, in which three maximum pressure ratios are 0.74892 (f1), 0.75892 (f2), and 0.76453 (f3).

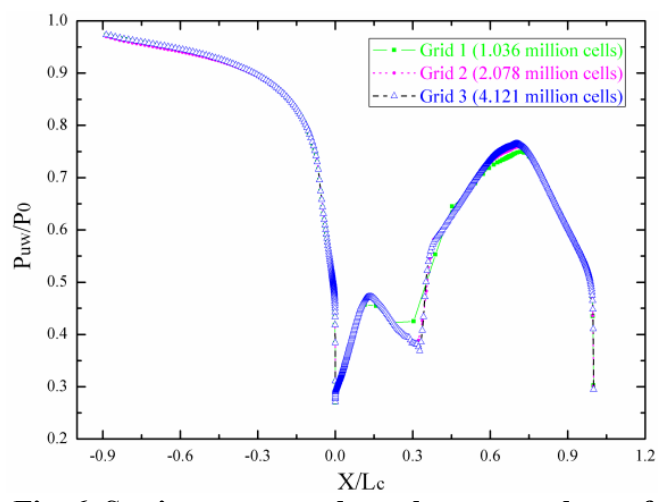

Fig. 6. Static pressures along the center-plane of the upper cavity wall for three grid systems.

The order of accuracy, q, is given by the following Eq. (12).

$q=\ln \left(\frac{f_{1}-f_{2}}{f_{2}-f_{3}}\right) / \ln (r)=0.83824893$

A safety factor Fs of 1.25 is utilized here (Wu et al. 2020b).

$G C I_{12}=\frac{F_{s}\left|\frac{f_{2}-f_{1}}{f_{2}}\right|}{r^{q}-1} * 100 \%=2.090521348 \%$

$G C I_{23}=\frac{F_{s}\left|\frac{f_{3}-f_{2}}{f_{3}}\right|}{r^{q}-1} * 100 \%=1.164176784 \%$

As a result,

$\frac{G C I_{12}}{r^{q \cdot G C I_{23}}}=1.004378947 \approx 1$

A nice solution is testified in the asymptotic range of convergence. As a result, grid 2 is the best option to run the current CFD work.

\section{RESULTS AND DISCUSSION}

\subsection{Validation}

The validation of the numerical manner is done against the existing experimental data from Flamm et al. (2006). The dimensions of the experimental nozzle are the same as the geometry used in the current CFD work, where Hut $=\mathrm{Hdt}=0.0292 \mathrm{~m}$, 
$\mathrm{Lc}=0.0762 \mathrm{~m}, \mathrm{~W}=0.1016 \mathrm{~m}, \theta 1=10^{\circ}$, and $\theta 2=$ $20^{\circ}$. Concerning the slot injector, its geometry dimensions are $\mathrm{D}=0.0005 \mathrm{~m}$ and $\mathrm{L}=0.1016 \mathrm{~m}$. The setup angle of the slot injector is $\lambda=150^{\circ}$. Operating conditions are given as follows: Stagnation pressures of the secondary stream and the mainstream are $607950 \mathrm{~Pa}$ and $405300 \mathrm{~Pa}$, severally. The standard ambient pressure is kept constant at $101325 \mathrm{~Pa}$. The stagnation temperature is $297.04 \mathrm{~K}$. Wang et al. (2019b) validated their CFD work using the shear stress transport k-omega (SST k- $\omega$ ), Realizable k-epsilon (k- $\varepsilon$ ), and SpalartAllmaras turbulence models and reported that the SST k- $\omega$ turbulence model shows the best consistency to match the experimental result. Consequently, the SST k- $\omega$ turbulence model is decided to verify the current CFD result. In Fig. 7, the static pressure along the center-plane of the upper cavity wall is compared with experimentally measured data, in which it has a nice match. In consequence, the SST $\mathrm{k}-\omega$ turbulence model is chosen.

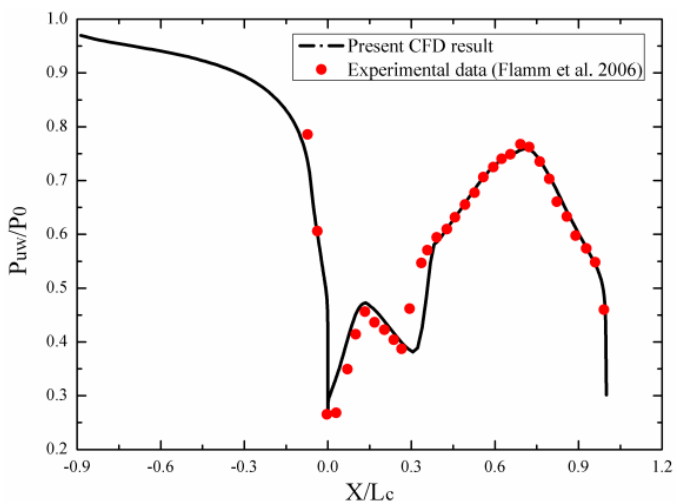

Fig. 7. Comparison of static pressures along the center-plane of the upper cavity wall (CFD vs. experiment).

\subsection{Impact of Nozzle Pressure Ratio}

The investigation of the DTNTVC performance for diverse NPR values needs to be carried through, which is of important significance since it may lead to an over-expanded or under-expanded jet. For increment in the NPR from 2 to 5 , an invariant injection pressure ratio (IPR) of 7.6 is used.

Fig. 8 depicts pitching angles and injection-tomainstream mass flow ratios for diverse levels of NPR. The pitching angle declines along with an increasing NPR. To be specific, the pitching angle apace decreases for NPR $<3$, whereas it tardily decays for NPR > 3. The injection-to-mainstream mass flow ratio quickly decays along with an increasing NPR. Fig. 9 presents 3-D streaklines for diverse values of NPR. All vortices are located at the cavity bottom. The development of these vortices that are limited by side-walls covers the full lateral direction. Moreover, these vortex structures are more similar for NPR $=2,4$, and 5 . For NPR $=2$, the volume of the vortex region is the largest. As a result, the squeeze effectiveness of these vortices acting on the mainstream is strongest to cause the largest pitching angle. Nevertheless, for $\mathrm{NPR}=3$, these vortices are more concentrated toward the central plane.

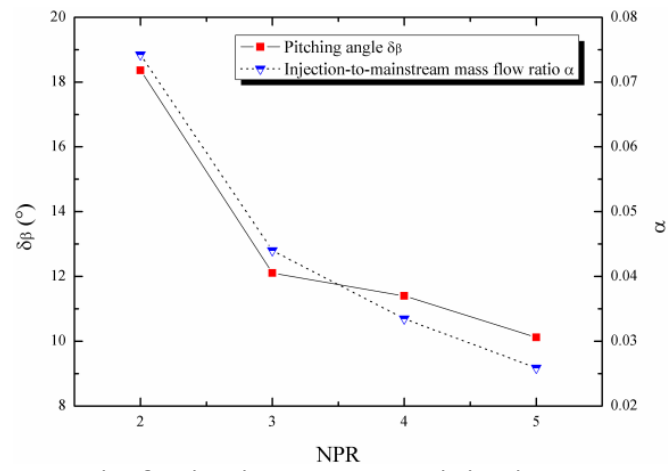

Fig. 8. Pitching angles and injection-to-

mainstream mass flow ratios for various levels of NPR.

Mach contours on the center-plane are portrayed in Fig. 10 for diverse levels of NPR. By referring to the skewed sonic-line, the degree of the mainstream deflection next to the DTN exit declines with an increasing NPR. Furthermore, the flow expansion outside the DTN exit transits from an overexpanded jet to an under-expanded jet. At the upstream nozzle throat, the enclosed sonic-line region takes up the entire concave cavity for NPR = 2. As the NPR enlarges, the enclosed sonic-line zone diminishes gradually due to the reduction of induced shocks. Fig. 11 shows the streaklines on the center-plane for diverse levels of NPR. The scale of the vortex region diminishes with an increasing NPR, resulting in a decay of the squeeze effect. In consequence, the extent of jet deflection weakens.

Systemic thrust ratios and systemic thrust efficiencies for diverse values of NPR are shown in Fig. 12. Systemic thrust ratio and thrust efficiency increase along with the increment of the NPR. The increasing thrust ratio is owing to the diminishing supersonic region in the concave cavity. Fewer inducted shocks form in this supersonic region, leading to fewer thrust losses. The increasing thrust efficiency is due to a faster decrement of the injection-to-mainstream mass flow rate in comparison to the decrement of the vectoring angle.

The Mach contours on various $\mathrm{X} / \mathrm{L}$ planes are shown in Fig. 13 for diverse levels of NPR. At X/L $=-0.25$, the subsonic flow is obtained because it is in the convergent part of the C-D nozzle. Since the mainstream momentum increases with an increasing NPR, the flow on this plane accelerates. On the plane of the upstream nozzle throat $(\mathrm{X} / \mathrm{L}=0)$, the supersonic flow is observed at the top and bottom positions, severally. Moreover, the area of the bottom supersonic region diminishes with an increasing NPR. Comprehensive comparisons on different sections of $\mathrm{X} / \mathrm{L}=0.25,0.5$, and 0.67 indicate remarkable variations on the vortex region. For NPR $=3$, the vortices are more intensive close to the center-plane, as previously explained in Fig. 
K. X. Wu et al. / JAFM, Vol. 14, No. 1, pp. 73-87, 2021.

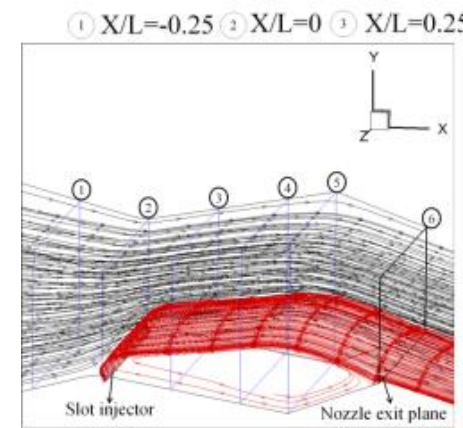

(a) $\mathrm{NPR}=2$

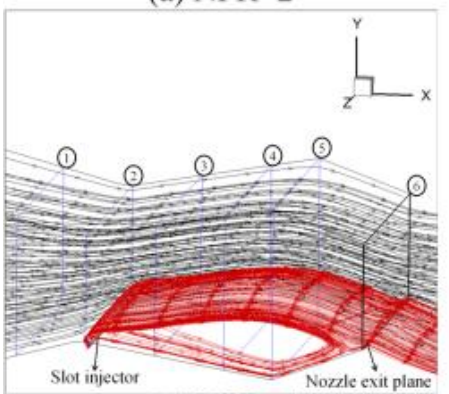

(c) $\mathrm{NPR}=4$

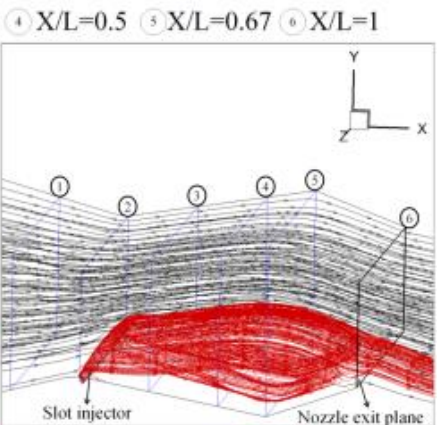

(b) $\mathrm{NPR}=3$

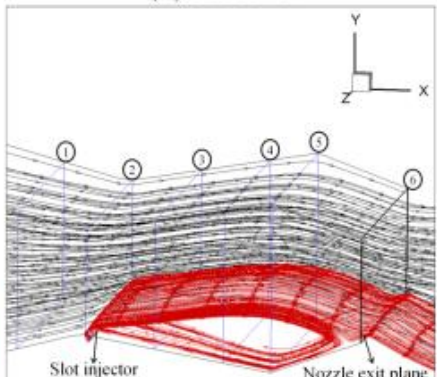

(d) $\mathrm{NPR}=5$

Fig. 9. 3-D streaklines for diverse values of $N P R$ (a) $N P R=2$; (b) $N P R=3$; (c) $N P R=4$; (d) $N P R=5$.

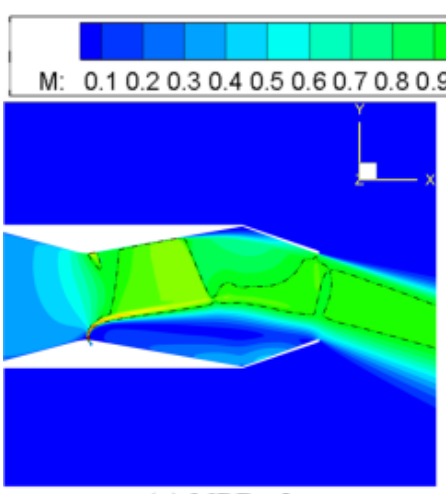

(a) $\mathrm{NPR}=2$

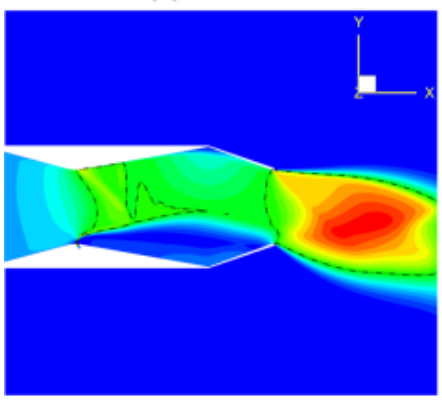

(c) $\mathrm{NPR}=4$

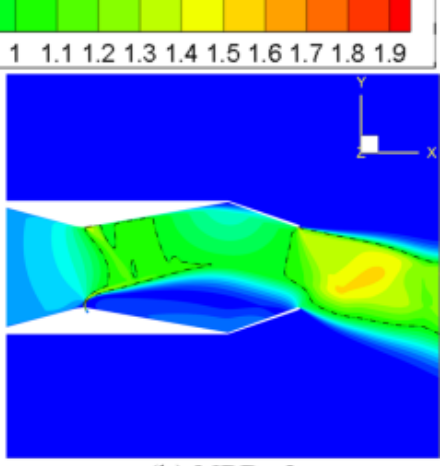

(b) NPR=3

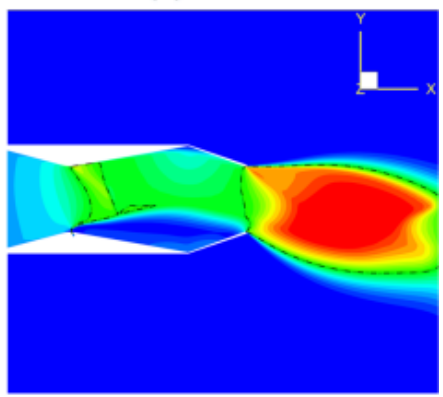

(d) $\mathrm{NPR}=5$

Fig. 10. Mach contours on the center-plane for different values of NPR (a) NPR = 2; (b) NPR = 3; (c) $\mathrm{NPR}=4$; (d) $\mathrm{NPR}=5$.

9. The supersonic region above the vortex zone diminishes along with the increasing NPR because of the diminishing induced shock. At the DTN exit $(\mathrm{X} / \mathrm{L}=1)$, the area of the supersonic region initially increases then reduces along with an increasing NPR. Besides, one can see that the 3-D flow-field is asymmetric using the center-plane as the basic reference. It is owing to the impacts of the subsonic vortices that have 3-D direction inconstancy and randomness. In consequence, a half domain with the symmetry boundary can not predict entire flowfield features precisely. The full domain is necessary to catch the 3-D rectangular DTNTVC flow-field characteristics. 


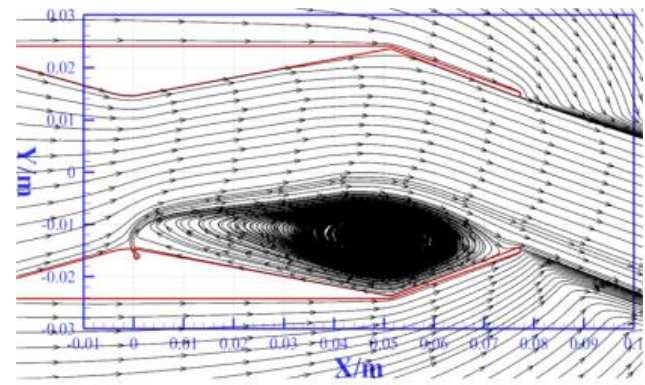

(a) $\mathrm{NPR}=2$

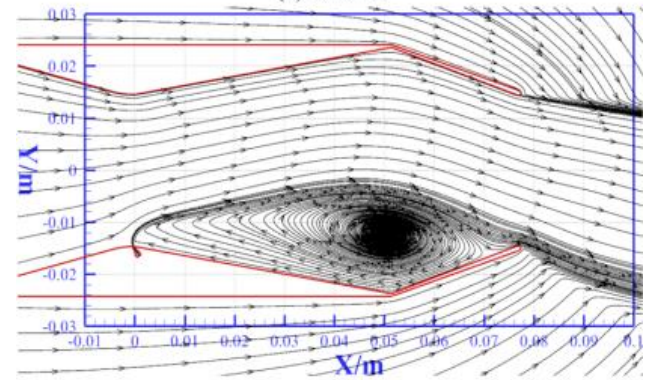

(b) $\mathrm{NPR}=3$

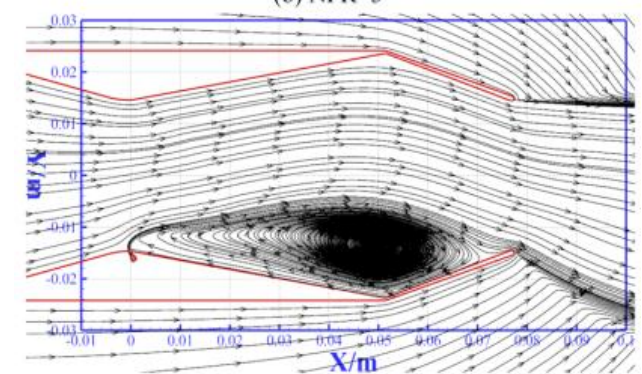

(c) $\mathrm{NPR}=4$

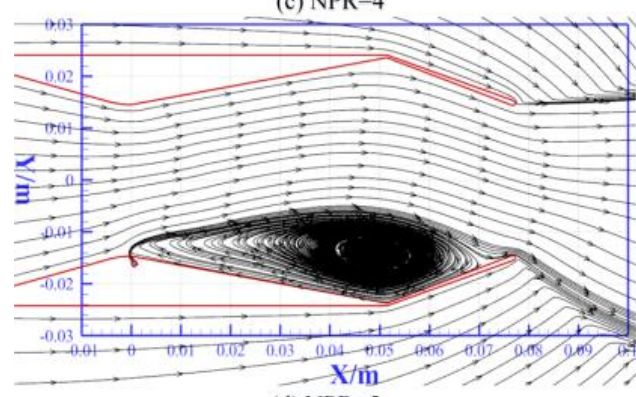

(d) $\mathrm{NPR}=5$

Fig. 11. Streaklines on the center-plane for diverse values of NPR (a) $\mathrm{NPR}=2$; (b) $\mathrm{NPR}=3$; (c) $\mathrm{NPR}=4$; (d) $\mathrm{NPR}=5$.

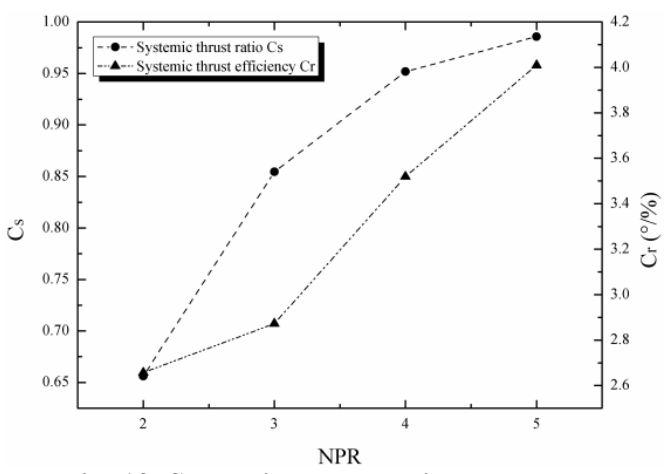

Fig. 12. Systemic thrust ratios and thrust efficiencies for diverse levels of NPR.

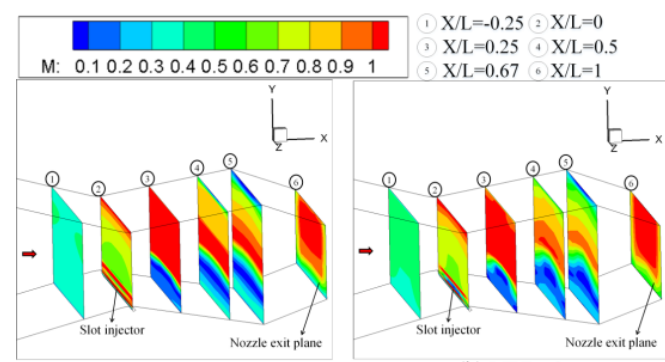

(a) $\mathrm{NPR}=2$

(b) $\mathrm{NPR}=3$

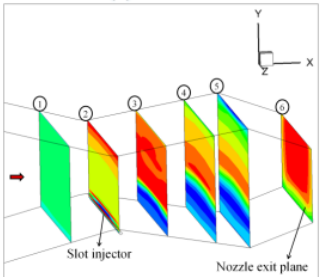

(c) $\mathrm{NPR}=4$

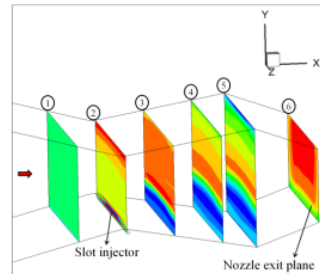

(d) $\mathrm{NPR}=5$
Fig. 13. Mach contours on various $X / L$ planes for diverse levels of NPR (a) $N P R=2$; (b) $N P R=3$;

(c) $\mathrm{NPR}=4$; (d) $\mathrm{NPR}=5$.

\subsection{Impact of the Injection-to-Mainstream Momentum Flux Ratio}

The internal flow of DTN is investigated for diverse injection-to-mainstream momentum flux ratios at NPR $=4$. The control of the injection-tomainstream momentum flux ratio is carried out by changing the injector pressure. Six different injection-to-mainstream momentum flux ratios are examined.

Fig. 14 shows the 3-D streaklines for diverse injection-to-mainstream momentum flux ratios. As different high-pressure secondary streams discharge from the slot exit, they start to squeeze and compel the mainstream to redirect and move downstream toward the upper divergent part of the concave cavity. The flow separation region that a series of vortices are rolled up occurs at the cavity bottom, owing to the powerful mainstream entrainment. Compare these six figures and you will see that the vortex occurs and develops along the full lateral direction, whereas it has to be limited by the sidewalls. Its volume gradually enlarges with an increasing injection-to-mainstream momentum flux ratio. Consequently, the squeeze effect of the vortex acting on the mainstream becomes more severe and further deepens the degree of mainstream deflection.

Fig. 15 shows pitching angles for diverse injectionto-mainstream momentum flux ratios. The pitching angle keeps increasing along with an increment of the injection-to-mainstream momentum flux ratio. At $\mathrm{J}<2.41$, the pitching angle quickly enlarges with an increasing injection-to-mainstream momentum flux ratio. For $\mathrm{J}>2.41$, although the pitching angle still enlarges along with an increasing injection-tomainstream momentum flux ratio, its growth rate decays. It is affected by the choking condition of the slot. While the slot does not choke, the fast increase of the mass flow rate of the secondary stream results in the quick growth of the vectoring 


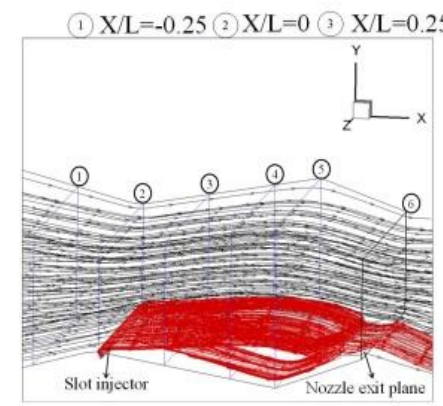

(a) $\mathrm{J}=0.43$

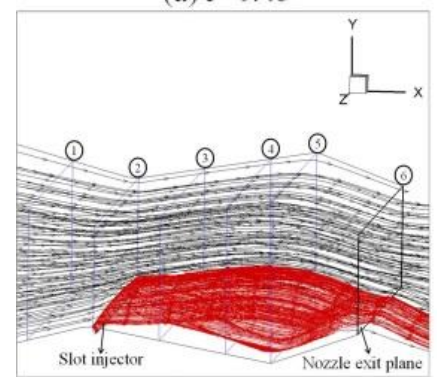

(c) $\mathrm{J}=1.58$

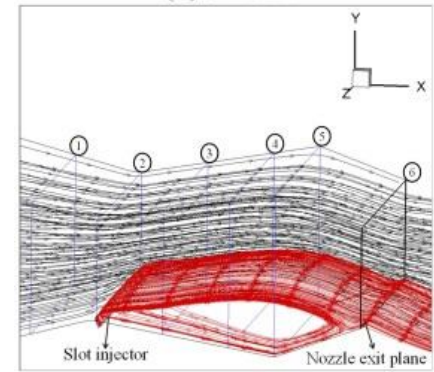

(e) $\mathrm{J}=2.41$

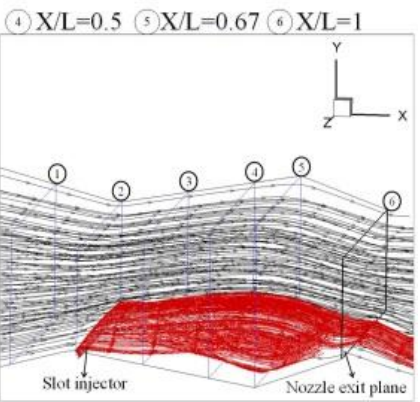

(b) $\mathrm{J}=0.98$

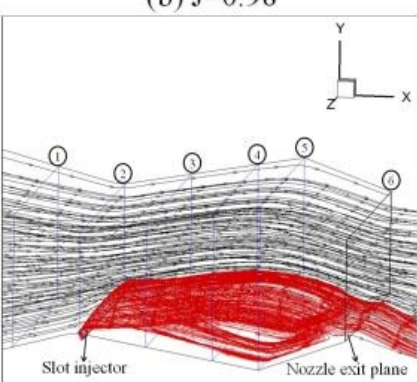

(d) $\mathrm{J}=2.01$

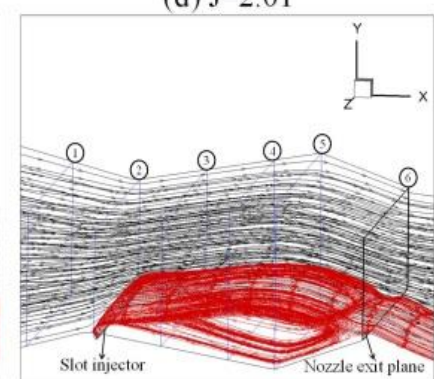

(f) $\mathrm{J}=\mathbf{2 . 7 6}$

Fig. 14. 3-D streaklines for different injection-to-mainstream momentum flux ratios (a) $\mathbf{J}=\mathbf{0 . 4 3}$; (b) $\mathbf{J}=$ 0.98; (c) $\mathrm{J}=1.58$; (d) $\mathrm{J}=\mathbf{2 . 0 1}$; (e) $\mathrm{J}=\mathbf{2 . 4 1}$; (f) $\mathrm{J}=\mathbf{2 . 7 6}$.

angle. At $\mathbf{J}>=2.41$, the slot injector is choked. Either injection-to-mainstream momentum flux ratio or pitching angle enlarges slowly.

Fig. 16 shows Mach contours on the center-plane for diverse injection-to-mainstream momentum flux ratios (Noting: the sonic-line is marked with the black dash-dot line). When the injection-tomainstream momentum flux ratio enlarges, the skewed degree of sonic-line at the downstream throat of DTN deepens. Near the upstream throat, the enclosed sonic-line zone continuously enlarges with an increasing injection-to-mainstream momentum flux ratio. At $\mathrm{J}=0.43$ and 0.98 , a small supersonic region appears next to the upper junction part of the convergent-divergent nozzle. As the injection-to-mainstream momentum flux ratio is $\mathrm{J}=$ 1.58 , the area of the small supersonic region enlarges but also one more tiny supersonic zone is observed near the slot exit. When the injection-tomainstream momentum flux ratio reaches $\mathrm{J}=2.01$, the above two independent supersonic regions merge into a larger supersonic region. For $\mathbf{J}>2.01$, the area of this larger supersonic zone continuously enlarges. All these above supersonic regions contain various induced shocks owing to the interactions between the high-pressure secondary stream and the mainstream. Fig. 17 depicts the streaklines on the center-plane for diverse injection-to-mainstream momentum flux ratios. A set of vortices form at the bottom of the concave cavity due to the strong mainstream entrainment. As the injection-tomainstream momentum flux ratio enlarges, the scale of the vortex region enlarges. Then, the squeeze effect of these vortices on the mainstream enhances.

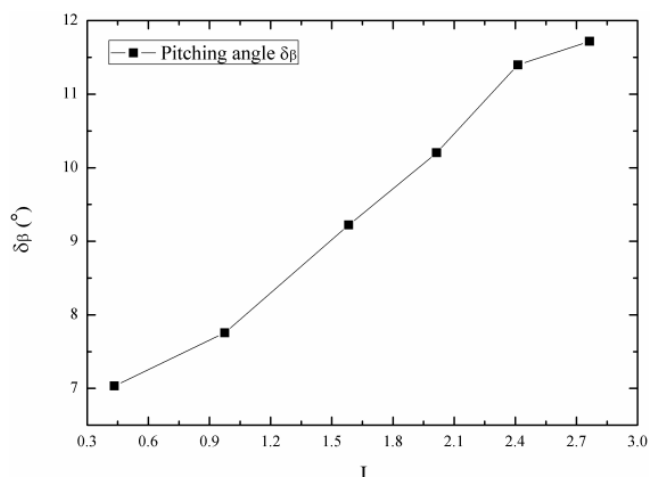

Fig. 15. Pitching angles for diverse injection-tomainstream momentum flux ratios. 


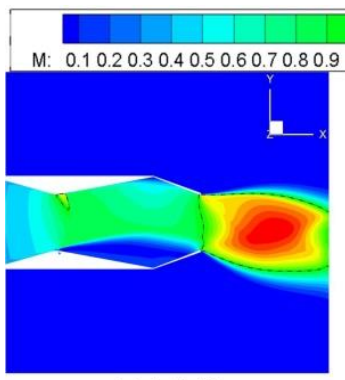

(a) $\mathrm{J}=0.43$

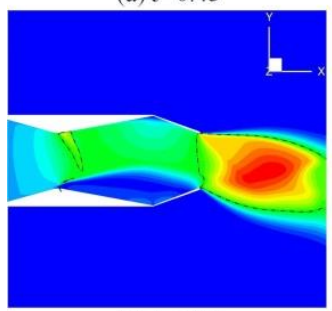

(c) $\mathrm{J}=1.58$

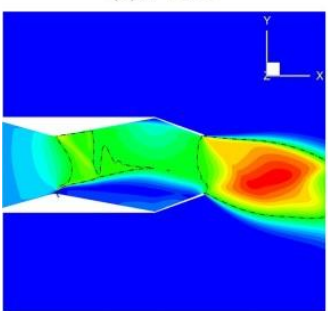

(e) $\mathrm{J}=2.41$

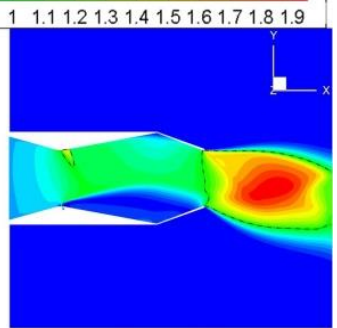

(b) $\mathrm{J}=0.98$

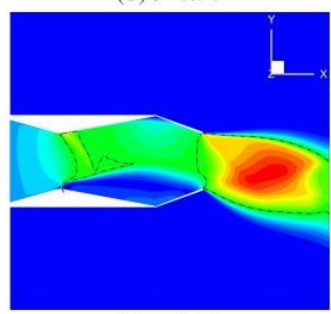

(d) $\mathrm{J}=2.01$

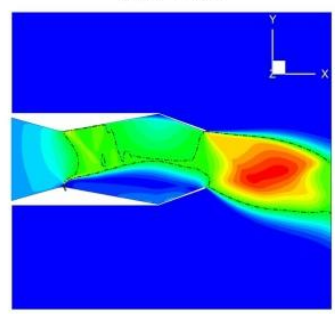

(f) $\mathrm{J}=2.76$
Fig. 16. Mach contours on the center-plane for different injection-to-mainstream momentum flux ratios (a) $\mathrm{J}=0.43$; (b) $\mathrm{J}=0.98$; (c) $\mathrm{J}=1.58$; (d) $\mathrm{J}=2.01$; (e) $\mathrm{J}=2.41$; (f) $\mathrm{J}=\mathbf{2 . 7 6}$.

Fig. 18 depicts systemic thrust ratios and thrust efficiencies for diverse injection-to-mainstream momentum flux ratios. Both systemic thrust ratio and thrust efficiency decline along with an increasing injection-to-mainstream momentum flux ratio. The declining systemic thrust ratio is due to the expanded sonic-line zone in the concave cavity. More induced shocks form in this zone resulting in more thrust losses. The decreasing thrust efficiency is mainly affected by the injection-to-mainstream mass flow ratio.

Fig. 19 presents the Mach contours on six different $\mathrm{X} / \mathrm{L}$ planes $(\mathrm{X} / \mathrm{L}=-0.25,0,0.25,0.5,0.67$, and 1$)$ for diverse injection-to-mainstream momentum flux ratios. The 3-D flow asymmetrically develops utilizing the center-plane as the basic reference. On the plane of $\mathrm{X} / \mathrm{L}=-0.25$, the fluid is subsonic. As the injection-to-mainstream momentum flux ratio increases, the size of the lower Mach number region enlarges. Because the stronger secondary stream has a larger deceleration effect on the upstream subsonic flow. At the location of the upstream throat $(\mathrm{X} / \mathrm{L}=0)$, the supersonic flow region appears on this plane. For $\mathrm{J}=0.43$, the supersonic flow region only occurs on the top portion, owing to the occurrence of induced shocks. For $\mathrm{J}>=0.98$, one more supersonic region appears next to the slot exit. According to three planes of $\mathrm{X} / \mathrm{L}=0.25,0.5$, and 0.67 , the shape and scale of the vortex zone at the cavity bottom can be distinguished. The volume of the whole vortex region gradually enlarges with an increasing injection-to-mainstream momentum flux ratio. Concerning the exit plane of DTN $(\mathrm{X} / \mathrm{L}=1)$, the area of the supersonic zone enlarges along with an enlarging injection-to-mainstream momentum flux ratio.

\subsection{Impact of the Setup Angle of the Slot Injector}

Seven angles of the slot injection syringe are considered, involving $30^{\circ}, 45^{\circ}, 60^{\circ}, 90^{\circ}, 120^{\circ}$, $135^{\circ}$, and $150^{\circ}$, as shown in Fig. 20. All present CFD work is performed for NPR $=4$ and IPR $=7.6$.

The 3-D streaklines in the DTN flow-field are depicted in Fig. 21 for diverse setup angles of the slot injector. For $\lambda<120^{\circ}$, the volume of these vortices enlarges along with an enlarging setup angle of the slot injector. For $\lambda>120^{\circ}$, its volume diminishes. Fig. 22 shows Mach contours on the center-plane for diverse setup angles of the slot injector. The sonic-line skewing enlarges along with an increasing setup angle for $\lambda<120^{\circ}$, whereas it starts to decline for $\lambda>120^{\circ}$. At the neighborhood of the upstream throat, the enclosed sonic-line region enlarges along with an increasing setup angle of the slot injector at $\lambda<120^{\circ}$; however, its area diminishes with a further increase of the setup angle. When the setup angle is less than $60^{\circ}$, two independent supersonic regions with small areas appear next to the upper junction of the conventional C-D nozzle and the slot exit. While the setup angle exceeds $60^{\circ}$, these two small independent supersonic regions merge into a larger supersonic zone.

Fig. 23 depicts pitching angles under diverse setup angles of the slot injector. The pitching angle rapidly increases along with the rise of the setup angle at $\lambda<120^{\circ}$, due to the enhanced squeeze effect of the enlarged vortex region. At $\lambda>120^{\circ}$, the pitching angle slowly declines along with an increment of the setup angle. Fig. 24 presents the streaklines on the center-plane for diverse setup angles of the slot injection syringe. As the setup angle of the slot injector is less than $120^{\circ}$, the scale of the vortex region enlarges along with an increment of the setup angle. When the setup angle exceeds $120^{\circ}$, the size of the vortex zone slightly diminishes along with the increasing setup angle in comparison to that of $\lambda=120^{\circ}$.

Fig. 25 shows the systemic thrust ratios and thrust efficiencies under diverse setup angles of the slot injection syringe. The systemic thrust ratio quickly decays along with an increment of the setup angle at $\lambda<120^{\circ}$. Because more induced shock waves cause higher thrust losses. The systemic thrust ratio decays and arrives at the lowest level at $\lambda=120^{\circ}$, then it follows the increase of the setup angle. Although the pitching angle declines for $\lambda>120^{\circ}$ compared to that of $\lambda=120^{\circ}$, a fast decrease of the mass flow rate of the secondary flow plays the main role to lead to the faster increment of the systemic thrust efficiency. 


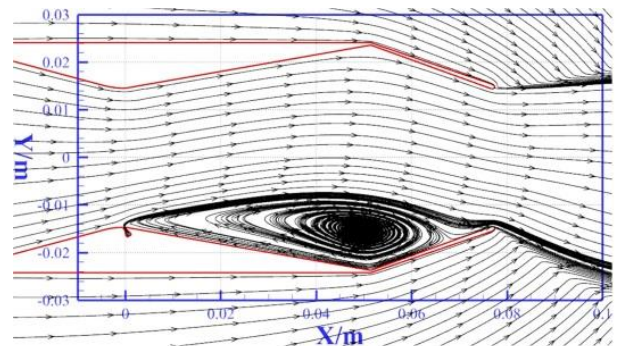

(a) $\mathrm{J}=0.43$

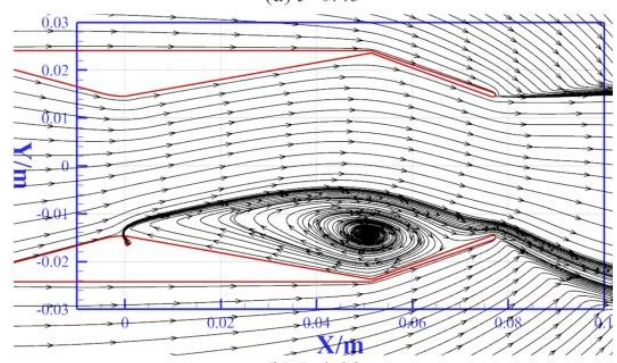

(b) $\mathrm{J}=0.98$

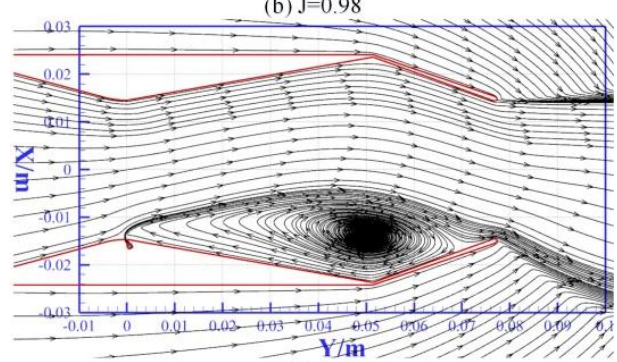

(c) $\mathrm{J}=1.58$

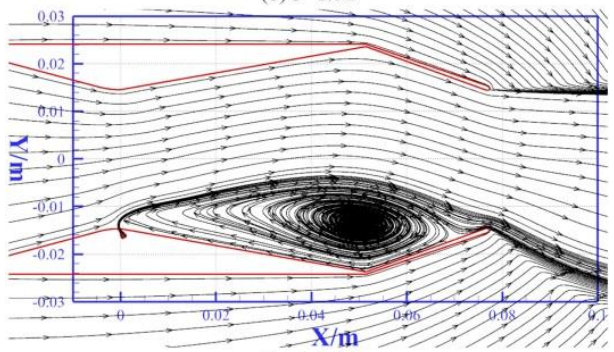

(d) $\mathrm{J}=2.01$

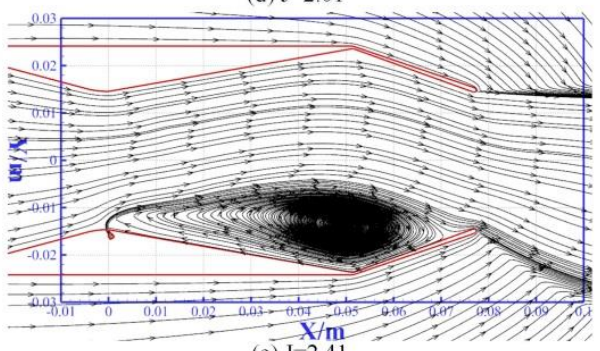

(e) $\mathrm{J}=2.41$

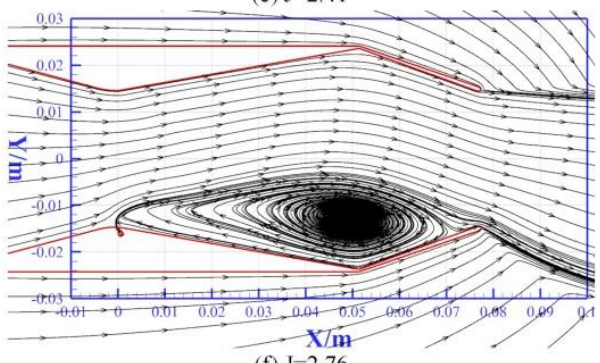

(f) $\mathrm{J}=2.76$

Fig. 17. Streaklines on the center-plane for diverse injection-to-mainstream momentum flux ratios (a) $\mathrm{J}=0.43$; (b) $\mathrm{J}=0.98$; (c) $\mathrm{J}=1.58$; (d) $\mathrm{J}$ $=2.01$; (e) $\mathrm{J}=2.41$; (f) $\mathrm{J}=2.76$.

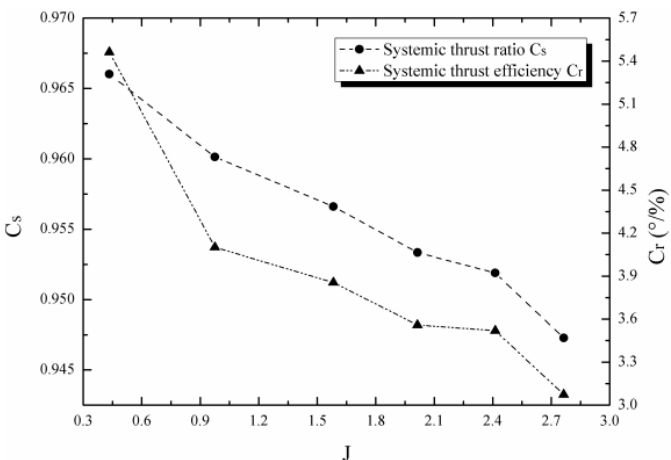

Fig. 18. Systemic thrust ratios and thrust efficiencies for diverse injection-to-mainstream momentum flux ratios.

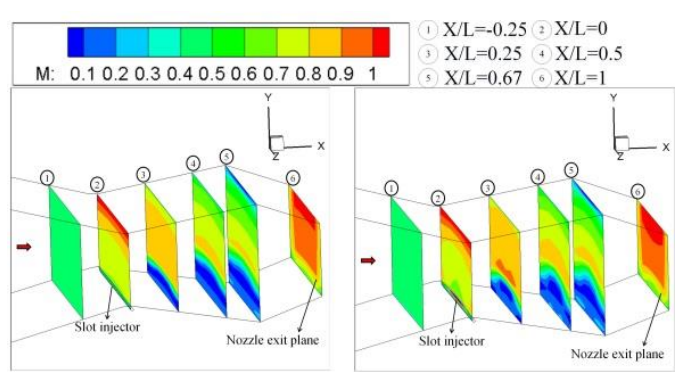

(a) $\mathrm{J}=0.43$

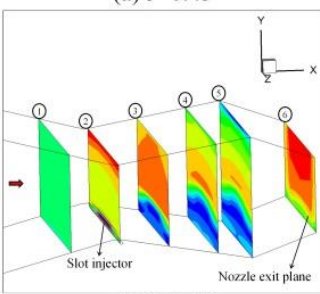

(c) $\mathrm{J}=1.58$

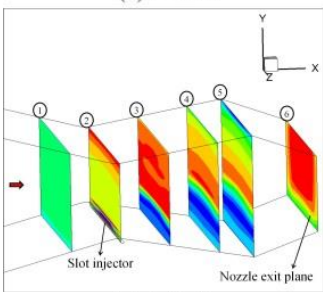

(e) $\mathrm{J}=2.41$ (b) $\mathrm{J}=0.98$

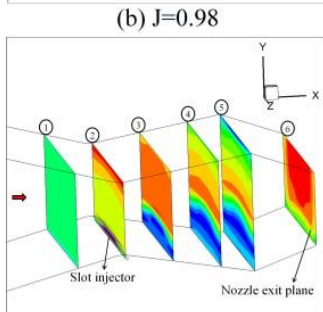

(d) $\mathrm{J}=2.01$

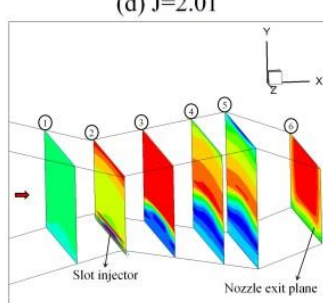

(f) $\mathrm{J}=2.76$
Fig. 19. Mach contours on different $X / L$ planes for diverse injection-to-mainstream momentum flux ratios (a) $\mathrm{J}=\mathbf{0 . 4 3}$; (b) $\mathrm{J}=\mathbf{0 . 9 8}$; (c) $\mathrm{J}=\mathbf{1 . 5 8}$; (d) $\mathrm{J}=2.01$; (e) $\mathrm{J}=2.41$; (f) $\mathrm{J}=\mathbf{2 . 7 6}$.

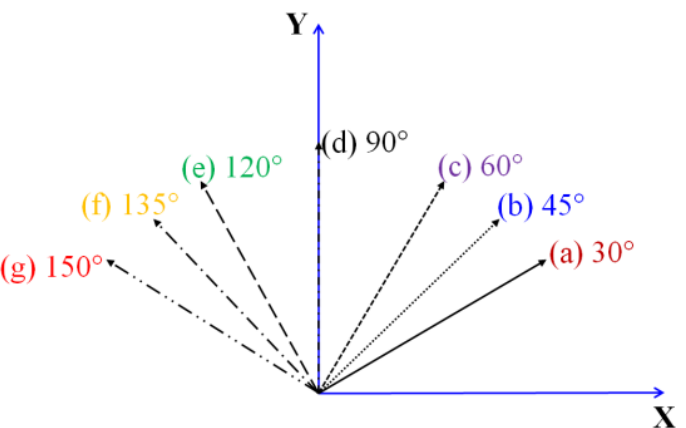

Fig. 20. Sketch for different setup angles of the slot injector (a) $\lambda=30^{\circ}$; (b) $\lambda=45^{\circ}$; (c) $\lambda=60^{\circ}$; (d) $\lambda=90^{\circ}$; (e) $\lambda=120^{\circ}$; (f) $\lambda=135^{\circ}$; (g) $\lambda=150^{\circ}$. 


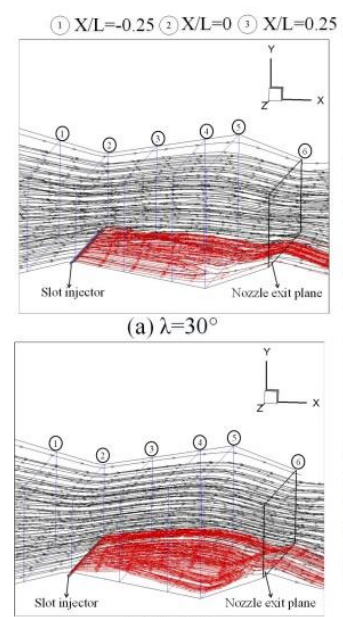

(c) $\lambda=60^{\circ}$

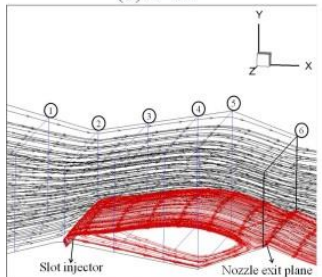

(e) $\lambda=120^{\circ}$

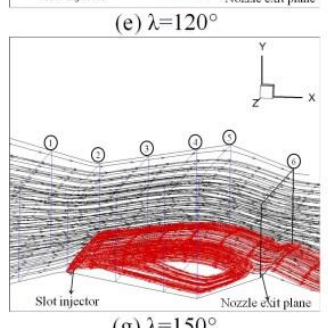

(g) $\lambda=150^{\circ}$

Fig. 21. 3-D streaklines for diverse setup angles of the slot injector (a) $\lambda=30^{\circ}$; (b) $\lambda=45^{\circ}$; (c) $\lambda=$ $60^{\circ}$; (d) $\lambda=90^{\circ}$; (e) $\lambda=120^{\circ}$; (f) $\lambda=135^{\circ}$; (g) $\lambda=$ $150^{\circ}$.

The Mach contours on six different X/L planes are exhibited in Fig. 26 for diverse setup angles of the slot injector. On the plane of $\mathrm{X} / \mathrm{L}=-0.25$, the subsonic flow is observed. At the upstream throat of the DTN $(X / L=0)$, the supersonic flow region appears next to the upper junction part of the C-D nozzle due to the occurrence of the induced shock. According to three sections of $\mathrm{X} / \mathrm{L}=0.25,0.5$, and 0.67 , the volume of the whole vortex region gradually enlarges with the increasing setup angle under the case of $\lambda<120^{\circ}$, as well as the area of the supersonic region above the vortex zone. For $\lambda>$ $120^{\circ}$, one can see that the volume of the vortex region diminishes slightly. About the nozzle exit plane $(X / L=1)$, the area of the supersonic region initially enlarges along with the increment of the setup angle for $\lambda<120^{\circ}$, then declines for $\lambda>120^{\circ}$. Meanwhile, the asymmetric flow properties in the cavity are proven for diverse setup angles of the slot injector.
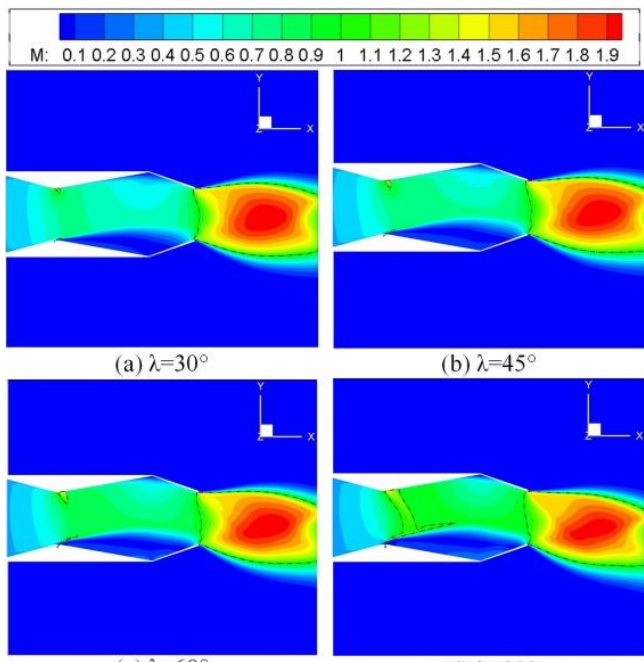

(c) $\lambda=60^{\circ}$

(d) $\lambda=90^{\circ}$

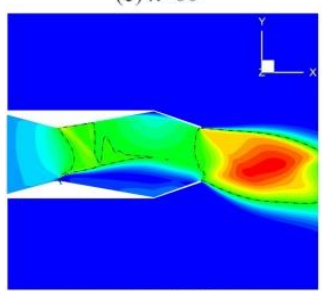

(e) $\lambda=120^{\circ}$
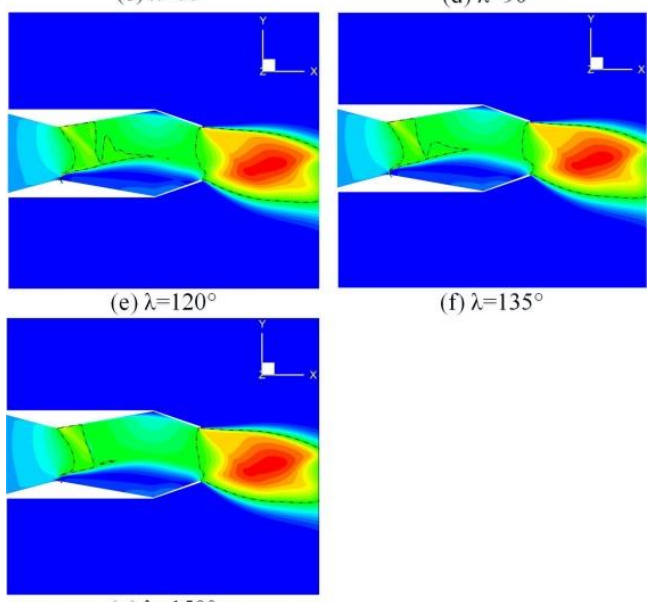

(f) $\lambda=135^{\circ}$

(g) $\lambda=150^{\circ}$

Fig. 22. Mach number contours on the centerplane for different setup angles of the slot injector (a) $\lambda=30^{\circ}$; (b) $\lambda=45^{\circ}$; (c) $\lambda=60^{\circ}$; (d) $\lambda$ $=90^{\circ}$; (e) $\lambda=120^{\circ}$; (f) $\lambda=135^{\circ}$; (g) $\lambda=150^{\circ}$.

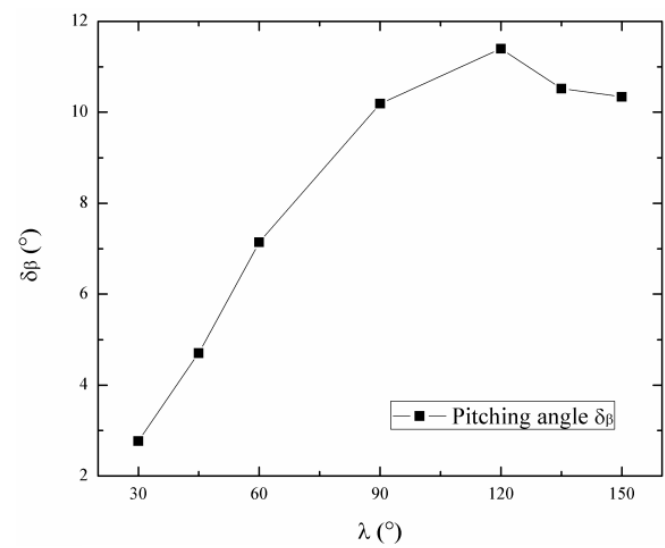

Fig. 23. Pitching angles for diverse setup angles of the slot injector. 
K. X. Wu et al. / JAFM, Vol. 14, No. 1, pp. 73-87, 2021.

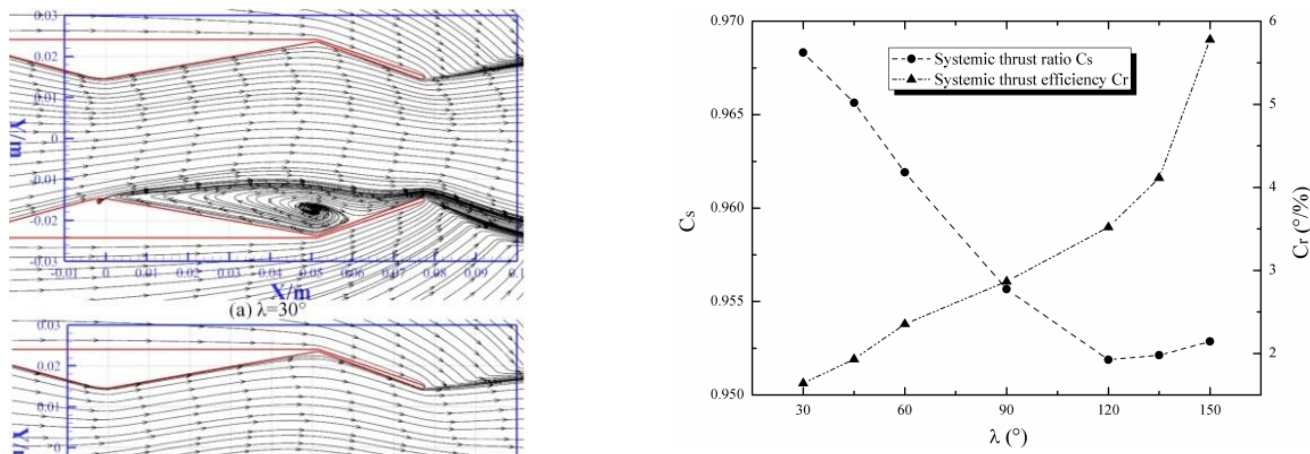

Fig. 25. Systemic thrust ratios and thrust efficiencies for diverse setup angles of the slot injector.

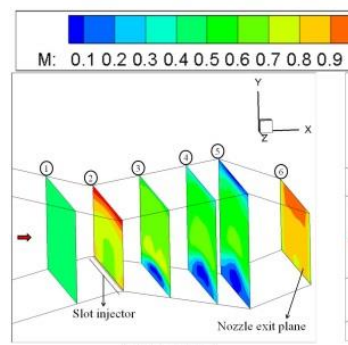

(a) $\lambda=30^{\circ}$

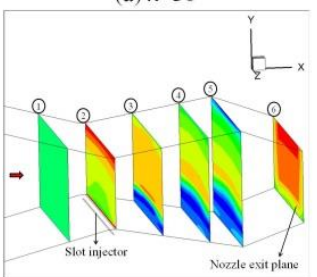

(c) $\lambda=60^{\circ}$

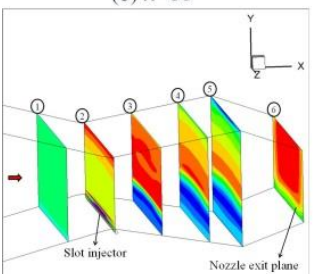

(e) $\lambda=120^{\circ}$

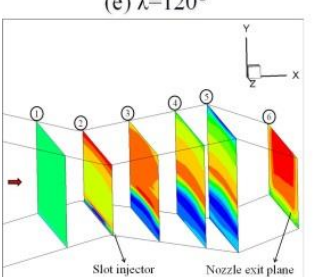

(g) $\lambda=150^{\circ}$

$\mathrm{L}=-0.25(2) \mathrm{X} / \mathrm{L}=0$ $\mathrm{X} / \mathrm{L}=0.25 \times \mathrm{X} / \mathrm{L}=0.5$

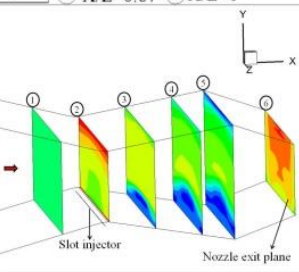

(b) $\lambda=45^{\circ}$

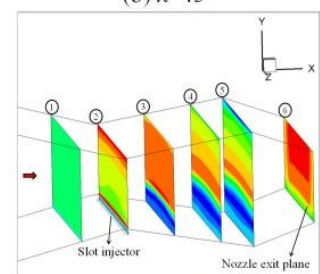

(d) $\lambda=90^{\circ}$

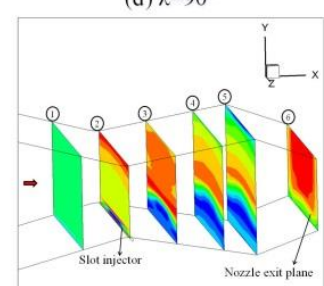

(f) $\lambda=135^{\circ}$

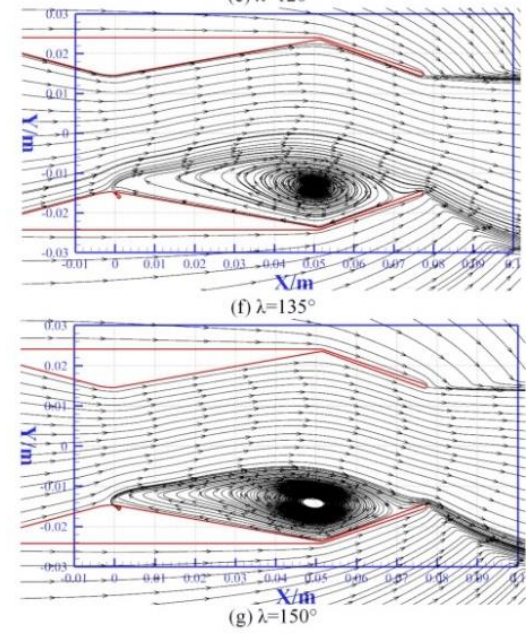

Fig. 24. Streaklines on the center-plane for different setup angles of the slot injector (a) $\lambda=$ $30^{\circ}$; (b) $\lambda=45^{\circ}$; (c) $\lambda=60^{\circ}$; (d) $\lambda=90^{\circ}$; (e) $\lambda=$ $120^{\circ}$; (f) $\lambda=135^{\circ}$; (g) $\lambda=150^{\circ}$.
Fig. 26. Mach contours on various $X / L$ planes for different setup angles of the slot injector (a) $\lambda=$ $30^{\circ}$; (b) $\lambda=45^{\circ}$; (c) $\lambda=60^{\circ}$; (d) $\lambda=90^{\circ}$; (e) $\lambda=$ $120^{\circ}$; (f) $\lambda=135^{\circ}$; (g) $\lambda=150^{\circ}$.

\section{CONClusion}

In a typical 3-D rectangular DTNTVC configuration, a set of parametric studies are carried through. The impacts of NPR, injection-tomainstream momentum flux ratio, and setup angle of the slot injection syringe are demonstrated. The 
feasibility of the current CFD work has been testified against existing experimental data. Excellent consistency is obtained. The main conclusions are summarized as follows.

The flow-field of the 3-D rectangular DTNTVC is not fully symmetric based on the reference of the center-plane. In consequence, a full domain is necessary to catch the flow-field characteristics accurately.

Under the circumstance of an invariant IPR level, the pitching angle keeps decreasing along with an increasing NPR. Systemic thrust ratio and thrust efficiency uniformly increase with the increment of the NPR. Compared to other FTVC techniques, the main goal of DTNTVC development is to achieve larger pitching angles and higher systemic thrust efficiencies. The secondary goal is to reduce the sacrifice of the systemic thrust ratio. Consequently,

NPR $=4$ is the most adaptive choice to achieve a comprehensive performance, which not only offers a large vectoring angle and systemic thrust efficiency but also avoids remarkable sacrifices of the systemic thrust ratio.

The pitching angle keeps increasing along with an increasing injection-to-mainstream momentum flux ratio. Systemic thrust ratio and thrust efficiency decline along with an increasing injection-tomainstream momentum flux ratio. For the injectionto-mainstream momentum flux ratio, the research goal is to find a suitable range of the injected momentum flux for obtaining considerable performance. As a result, a sonically choked flow at the injector exit is more adaptive to offer comprehensive performance. The injection-tomainstream momentum flux ratio of $\mathbf{J}=2.41$ is the optimal choice.

The setup angle of the slot injector affects the internal performance of the DTN significantly. Even though a prominent advantage of the largest pitching angle is gained for the case of $\lambda=120^{\circ}$, whereas a more comprehensive and attractive performance is testified for $\lambda=150^{\circ}$. As a result, the setup angle of the slot injector for $\lambda=150^{\circ}$ is adapted to achieve a comprehensive performance of the DTNTVC system.

\section{ACKNOWLEDGEMENTS}

This work was supported by the National Research Foundation of Korea (NRF) grant funded by the Korea government(MSIP) (No. NRF2016R1A2B3016436).

\section{REFERENCES}

Bellandi, E. G. and A. J. Slippey (2009). Preliminary analysis and design enhancements of a dual-throat FTV nozzle concept. In 39th AIAA fluid dynamics conference, 3900.

Das, S. S., J. C. Páscoa, M. Trancossi and A. Dumas (2016). Computational fluid dynamic study on a novel propulsive system: ACHEON and its integration with an unmanned aerial vehicle (UAV). Journal of Aerospace Engineering 29(1), 1-16.

Deere, K. A. (2003). Summary of fluidic thrust vectoring research conducted at NASA Langley Research Center. In 21st AIAA applied aerodynamics conference, 3800 .

Deere, K. A., J. D. Flamm, B. L. Berrier and S. K. Johnson (2007). Computational study of an axisymmetric dual throat fluidic thrust vectoring nozzle concept for supersonic aircraft application. In 43rd AIAA/ASME/SAE/ASEE joint propulsion conference \& exhibit, 5085.

Deere, K. A., B. L. Berrier, J. D. Flamm and S. K. Johnson (2003). Computational study of fluidic thrust vectoring using separation control in a nozzle. In 21st AIAA applied aerodynamics conference, 3803.

Deng, R. Y. and H. D. Kim (2015). A study on the thrust vector control using a bypass flow passage. Proceedings of the Institution of Mechanical Engineers, Part G: Journal of Aerospace Engineering 229(9), 1722-1729.

Ferlauto, M. and R. Marsilio (2009). Numerical simulation of fluidic thrust-vectoring. Journal of Aerospace Science, Technology and Systems 95(3), $1-8$

Flamm, J. D., K. A. Deere, B. L. Berrier and S. K. Johnson (2006). Design enhancements of the two-dimensional, dual throat fluidic thrust vectoring nozzle concept. In 3rd AIAA flow control conference, 3701.

Flamm, J. D., K. A. Deere, M. L. Mason, B. L. Berrier and S. K. Johnson (2007). Experimental study of an axisymmetric dual throat fluidic thrust vectoring nozzle for a supersonic aircraft application. In 43rd AIAA/ASME/SAE/ASEE joint propulsion conference \& exhibit, 5084.

Fleeman, E. L. (2006). Tactical missile design. Second Edition. AIAA.

Gu, R. and J. Xu (2015). Dynamic experimental investigations of a bypass dual throat nozzle. Journal of Engineering for Gas Turbines and Power 137, 1-6.

Gu, R., J. Xu and S. Guo (2014). Experimental and numerical investigations of a bypass dual throat nozzle. Journal of Engineering for Gas Turbines and Power, 136, 1-6.

Heo, J. Y. and H. G. Sung (2012). Fluidic thrustvector control of supersonic jet using coflow injection. Journal of Propulsion and Power 28(4), 858-861.

Kim, Y. C., T. S. Roh, H. Huh and H. J. Lee (2020). Study on the combined effect of various injection conditions on the drag reduction by a counter-flow jet in supersonic flow. Aerospace Science and Technology 98, 105580. 
Lin, Z., X. W. Sun, T. C. Yu, Y. F. Zhang, Y. Li and Z. C. Zhu (2020). Gas-solid two-phase flow and erosion calculation of gate valve based on the CFD-DEM model. Powder Technology 366, 395-407.

Raman, S. K., K. X. Wu, T. H. Kim and H. D. Kim (2020). Effects of flap on the reentry aerodynamics of a blunt cone in the supersonic flow. International Journal of Mechanical Sciences 176, 105396.

Roache, P. J. (1994). Perspective: a method for uniform reporting of grid refinement studies. Journal of Fluid Engineering 116, 405-413.

Shin, C. S., H. D. Kim, T. Setoguchi and S. Matsuo (2010). A computational study of thrust vectoring control using dual throat nozzle. Journal of Thermal Science 19, 486-490.

Sutton, G. P. and O. Biblarz (2001). Rocket propulsion elements, Seventh Edition. Wiley.

Tao, J. Y., Z. Lin, C. J. Ma, J. H. Ye, Z. C. Zhu, Y. $\mathrm{Li}$ and W. Mao (2020). An experimental and numerical study of regulating performance and flow loss in a v-port ball valve. Journal of Fluid Engineering 142(2), 172-182.

Vignesh, R. P. S., S. Das and H. D. Kim (2016). Influence of vortex generator on cylindrical protrusion aerodynamics at various Mach numbers. Aerospace Science and Technology 58: $267-274$

Vignesh, R. P. S., T. H. Kim and H. D. Kim (2019). Numerical study on shock train characteristics in divergent channels. Journal of Applied Fluid Mechanics 13(4): 267-274.

Wang, Y. S., J. L. Xu and S. Huang (2017). Study of starting problem of axisymmetric divergent dual throat nozzle. Journal of Engineering of Gas Turbines and Power 139, 1-13.

Wang, Y. S., J. L. Xu, S. Huang, Y. C. Lin and J. J. Jiang (2019a). Computational study of axisymmetric divergent bypass dual throat nozzle. Aerospace Science and Technology 86, 177-190.

Wang, Y. S., J. L. Xu, S. Huang, Y. C. Lin and J. J. Jiang (2019b). Experimental and numerical investigation of an axisymmetric divergent dual throat nozzle. Proceedings of the Institution of Mechanical Engineers, Part G: Journal of Aerospace Engineering 234(3), 563-572.

Wu, K. X. and H. D. Kim (2019a). Fluidic thrust vector control using shock wave concept. Journal of the Korean Society of Propulsion Engineers 23(4), 10-20.

Wu, K. X. and H. D. Kim (2019b). Numerical study on the shock vector control in a rectangular supersonic nozzle. Proceedings of the Institution of Mechanical Engineers, Part G: Journal of Aerospace Engineering 233(13), 4943-4965.

Wu, K. X. and H. D. Kim (2019c). Study on fluidic thrust vector control based on dual-throat concept. Journal of the Korean Society of Propulsion Engineers 23(1), 24-32.

Wu, K. X., G. Zhang, T. H. Kim and H. D. Kim (2020a). Numerical parametric study on threedimensional rectangular counter-flow thrust vectoring control. Proceedings of the Institution of Mechanical Engineers, Part G: Journal of Aerospace Engineering.

Wu, K. X., H. D. Kim and Y. Z. Jin (2018). Fluidic thrust vector control based on counter-flow concept. Proceedings of the Institution of Mechanical Engineers, Part G: Journal of Aerospace Engineering 233(4): 1412-1422.

Wu, K. X., S. K. Raman, R. P. S. Vignesh, G. Zhang and H. D. Kim. (2020b). Effect of the wall temperature on Mach stem transformation in pseudo-steady shock wave reflections. International Journal of Heat and Mass Transfer 147: 118927.

Wu, K. X., T. H. Kim and H. D. Kim. (2020c). Theoretical and numerical analyses of aerodynamic characteristics on shock vector control. Journal of Aerospace Engineering 33(5), 1-26.

Wu, K. X., T. H. Kim, J. K. James and H. D. Kim. (2020d). Assessment of the counter-flow thrust vector control in a three-dimensional rectangular nozzle. Journal of the Korean Society of Propulsion Engineers 24(1), 34-46.

Wu, K. X., Y. Z. Jin and H. D. Kim (2019). Hysteresis behaviors in counter-flow thrust vector control. Journal of Aerospace Engineering 32(4), 1-9.

Yagle, P. J., D. N. Miller, K. B. Ginn and J. W. Hamstra (2001). Demonstration of fluidic throat skewing for thrust vectoring in structurally fixed nozzles. Journal of Engineering of Gas Turbines and Power 123(3), 502-507. 